\title{
Ethyl-3,4-dephostatin Inhibits Dual-specificity phosphatase 22 (DUSP22) Activity
}

\author{
Huiyun Seo, Heemun Kim, and Sayeon Cho* \\ College of Pharmacy, Chung-Ang University, Seoul 156-756, Korea. *E-mail: sycho@cau.ac.kr \\ Received February 12, 2010, Accepted February 16, 2011
}

Key Words : DUSP22, Ethyl-3,4-dephostatin, PTP inhibitor

Protein phosphorylation is one of the major protein modification processes that play critical roles in a variety of biological processes, including gene expression, proliferation, differentiation, cell cycle arrest, and apoptosis., ${ }^{1,2}$ Protein phosphorylation usually occurs in serine, threonine, or tyrosine residues and is processed by protein kinases. Of those protein kinases, extracellular signal-regulated kinase (ERK), p38, and $c$-Jun $N$-terminal kinase (JNK) that are members of mitogen-activated protein kinases (MAPK) family, have diverse roles in cell proliferation, cell survival, cell death, differentiation, development, immune function, gene expression, and other intracellular events. ${ }^{3-5}$ Therefore, regulation of those MAPK family members results in diverse outcome of cell fate. Many of protein tyrosine phosphatases (PTPs) control the activation of MAPKs through dephosphorylation of phosphoserine, phosphothreonine, or phosphotyrosine residues that are located on the activation loop of MAPKs. The human genome contains 107 PTP genes. ${ }^{6}$ Dualspecificity phosphatases (DUSPs) as a heterogeneous group of protein phosphatases belong to a subclass of PTP families and dephosphorylate both phosphotyrosine and phosphoserine/phosphothreonine residues. ${ }^{7}$

Many studies have demonstrated that the modulation of PTP enzymatic activity is involved in regulating diverse cellular biological functions and disease susceptibility. ${ }^{8}$ Thus, chemical compounds that regulate the activity of PTPs may have ability to be used as therapeutic reagents for diseases such as cancer, inflammation, and diabetes.

Ethyl-3,4-dephostatin is a stable synthetic analog of dephostatin and was originally identified as a potent inhibitor of PTP-1B and Src homology region 2 (SH2) domain-containing phosphatase 1 (SHP-1) $)^{9,10}$ (Fig. 1). Since ethyl-3,4dephostatin might regulate other phosphatases, we screened PTPs by performing in vitro phosphatase assays to identify phosphatases that are inhibited by ethyl-3,4-dephostatin. We found that DUSP22 activity was inhibited by ethyl-3,4dephostatin.

DUSP22 was treated with various concentrations of ethyl3,4-dephostatin and then its phosphatase activity was

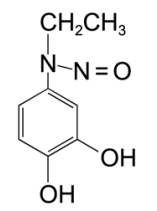

Figure 1. Chemical structure of Ethyl-3,4-dephostatin. measured. DUSP22 activity was decreased by ethyl-3,4dephostatin in a dose-dependent manner. An inhibition curve was plotted for DUSP22 and the half maximal inhibitory concentration $\left(\mathrm{IC}_{50}\right)$ was obtained. This result suggests that ethyl-3,4-dephostatin inhibits DUSP22 with $\mathrm{IC}_{50}$ of $3.06 \pm$ $0.07 \mu \mathrm{M}$ (Fig. 2(a)).

In subsequent experiments, kinetic analyses based on the Michaelis-Menten equation were performed with ethyl-3,4dephostatin and DUSP22 to provide experimental evidences for the mechanism of DUSP22 catalysis and for binding of
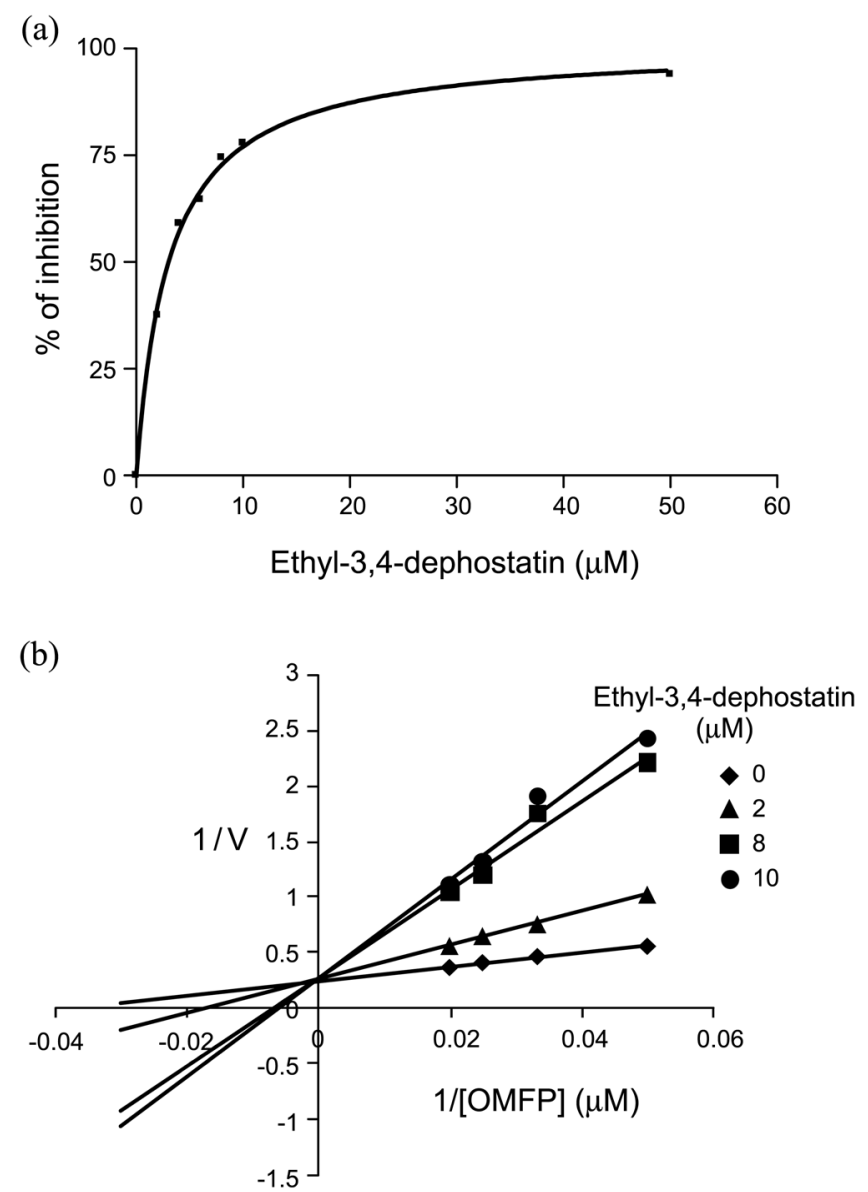

Figure 2. Inhibitory effect of Ethyl-3,4-dephostatin on DUSP22 and Kinetic analysis of DUSP22 inhibition by Ethyl-3,4-dephostatin. (a) DUSP22 were incubated with various concentrations of ethyl-3,4-dephostatin at $37^{\circ} \mathrm{C}$ for $30 \mathrm{~min}$. Fluorescence emission from the product was measured with a multiwell plate reader as described in Experimental section. (b) Lineweaver-Burk plots of DUSP22 generated from the reciprocal data. 
the inhibitor to the active site of the phosphatase. The $K_{m}$ value of DUSP22 for OMFP was $31.45 \pm 1.8 \mu \mathrm{M}$. Then, the Lineweaver-Burk plots show that the $K_{i}$ was $1.98 \mu \mathrm{M}$ (Fig. 2(b)). The results also show that the ethyl-3,4-dephostatin is a competitive inhibitor of DUSP22, suggesting that ethyl3,4-dephostatin inhibits the activity of DUSP22 through binding to the catalytic site.

Next, we searched for MAPKs that are regulated by DUSP22 in vivo. DUSP22 is similar to several other DUSPs. However, the intrinsic serine/threonine phosphatase activity of DUSP22 is still unclear in vivo. One of the reports showed that DUSP22 could dephosphorylate ERK in vitro and its expression has been found to suppress T cell antigen receptorinduced activation of ERK2 in Jurkat T cells. ${ }^{11}$ Another report suggested that DUSP22 could preferentially dephosphorylate and inactivate $\mathrm{JNK}$ and $\mathrm{p} 38$, but not ERK, in transfected COS-7 cells. $^{12}$ However, independent studies determined

(a)

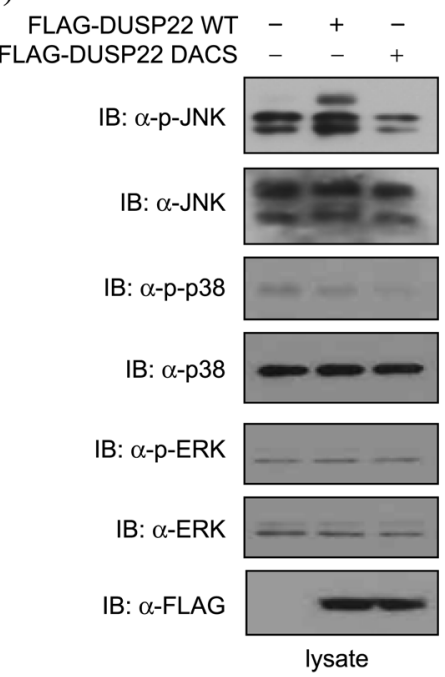

that DUSP22 selectively activates JNK in HEK 293 T cells ${ }^{13}$ and COS- 1 cells. ${ }^{14}$ To identify which MAPK is regulated by DUSP22, HEK 293 cells were transfected with FLAGDUSP22 WT or catalytically inactive double mutant DUSP22 DACS (D57A and C88S) expression plasmid. After $48 \mathrm{~h}$ of transfection, levels of phospho-JNK, phospho-ERK, and phospho-p38 were determined with Western blotting analysis. The phospho-JNK levels were increased by expression of DUPS22 WT in HEK 293 cells but not by the inactive DUPS22 mutant (Fig. 3(a)). However, levels of phosphoERK, and phospho-p38 were not significantly changed in the presence of DUSP22. These results suggest that DUSP22 induces JNK phosphorylation in HEK 293 cells and its phosphatase activity is required for JNK phosphorylation.

To confirm that ethyl-3,4-dephostatin negatively regulates DUSP22 phosphatase activity in vivo, HEK 293 cells were transiently transfected with FLAG-tagged DUSP22 WT or

(b)
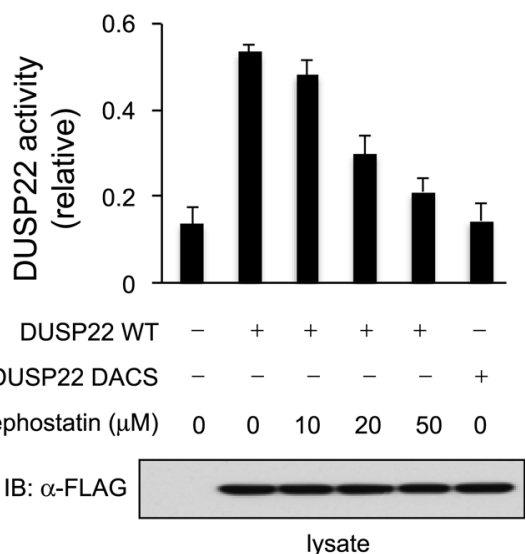

(c)

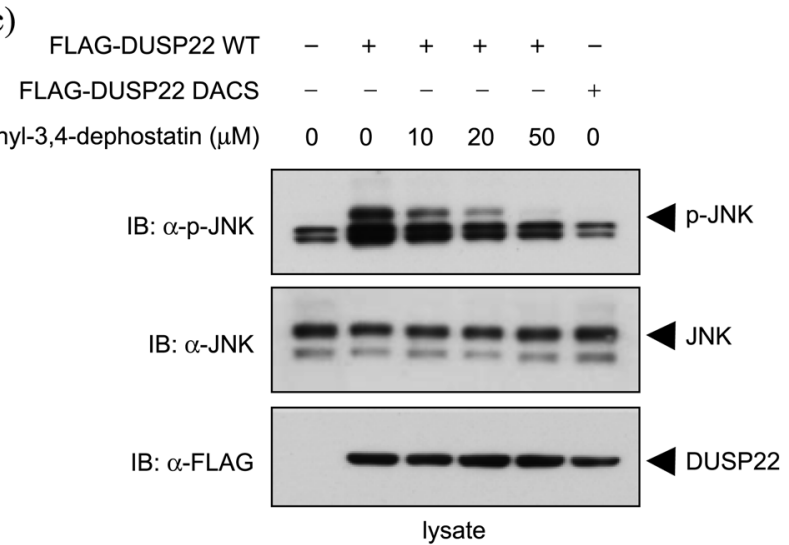

Figure 3. DUSP22 specifically activates JNK in HEK 293 cells and Ethyl-3,4-dephostatin inhibits DUSP22-dependent JNK activation. (a) HEK 293 cells were transfected with $1 \mu \mathrm{g}$ of DUSP22 WT or DACS expression plasmid. After $48 \mathrm{~h}$ of transfection, HEK 293 cells lysates were separated by SDS-PAGE and immunoblotted with appropriate antibodies. (b) HEK 293 cells were transfected with $1 \mu \mathrm{g}$ of DUSP22 WT or DACS expression plasmid. Transfected HEK 293 cells were pretreated with various concentrations of Ethyl-3,4-dephostatin (0, 10, 20 , or $50 \mu \mathrm{M}$ ) for $3 \mathrm{~h}$. FLAG-DUSP22 transfected cells were subjected to immunoprecipitation with anti-FLAG M2 agarose. Immunoprecipitates were assayed at $30{ }^{\circ} \mathrm{C}$ in $100 \mu \mathrm{L}$ of PTP reaction buffer. The amount of OMFP was determined by the change in absorbance at $490 \mathrm{~nm}$. Experiments were repeated three times with similar results. (c) HEK 293 cells were transfected with $1 \mu \mathrm{g}$ of DUSP22 WT or DACS expression plasmid. Transfected HEK 293 cells were pretreated with various concentrations of ethyl-3,4-dephostatin $(0,10$, 20 , or $50 \mu \mathrm{M}$ ) for $3 \mathrm{~h}$. Cell lysates were analyzed by immunoblotting with appropriate antibodies. 
mutant expression plasmid. Transfected HEK 293 cells were pretreated with $0-50 \mu \mathrm{M}$ of ethyl-3,4-dephostatin for $3 \mathrm{~h}$ before cells were lysed with PTP lysis buffer. DUSP22 was immunoprecipitated using anti-FLAG M2-agarose. Phosphatase activity of immunoprecipitated DUSP22 was then measured using OMFP as a substrate. Ethyl-3,4-dephostatin inhibits DUSP22 phosphatase activity in vivo (Fig. 3(b)). The results imply that ethyl-3,4-dephostatin transports into cells and inhibits DUSP22 activity.

Next, the in vivo effect of ethyl-3,4-dephostatin on DUSP22 activity were determined by measuring the phosphorylation status of endogenous JNK. HEK 293 cells were transfected with FLAG-DUSP22 expression plasmid, pretreated with various concentrations of ethyl-3,4-dephostatin for $3 \mathrm{~h}$, and then the levels of phospho-JNK were determined with Western blotting analysis. As shown in Figure 3(c), DUSP22mediated phosphorylation of JNK was significantly inhibited by ethyl-3,4-dephostatin. This result suggests that ethyl-3,4dephostatin effectively reduces DUSP22-activated JNK activity by inhibiting DUSP22 activity.

Early reports have shown that DUSP22 dephosphorylates several MAPKs. However, we found that DUSP22 has no phosphatase activity towards MAPK but induces phosphorylation of JNK in HEK 293 cells. In this study, we found that ethyl-3,4-dephostatin acts as an inhibitor of DUSP22. In conclusion, we verified that ethyl-3,4-dephostatin inhibits DUSP22 phosphatase activity and therefore reduces DUSP22dependent JNK activation.

\section{Experimental Section}

Cell Culture and Transfection. Human embryonic kidney (HEK) 293 cells were maintained at $37{ }^{\circ} \mathrm{C}$ in Dulbecco's modified Eagle's medium (DMEM, Invitrogen, Carlsbad California) supplemented with $10 \%$ fetal bovine serum (FBS, Invitrogen) and penicillin/streptomycin in the presence of $5 \% \mathrm{CO}_{2}$. For transient transfection, $1.4 \times 10^{6}$ cells were plated in each $60 \mathrm{~mm}$ cell culture plate, grown overnight, and transfected with DNA using LipofectAMINE (Invitrogen).

Plasmid Constructions. FLAG-tagged DUSP22 wild-type and DUSP22 DACS mutant were constructed in pcDNA3.1 (Invitrogen). His-tagged DUSP22 WT was constructed in pET28a plasmid (Novagen, Darmstadt, Germany) for protein expression in Escherichia coli.

Reagents and Antibodies. Anti-phospho-JNK (specific for phospho-Thr183 and phospho-Tyr185), anti-p38, antiphospho-p38 (Thr-180/Tyr-182), anti-ERK1/2, and antiphospho-ERK1/2 (Thr-202/Tyr-204) antibodies were purchased from Cell Signaling Technology (Danvers, MA). AntiJNK antibody was from Santa Cruz Biotechnology (Santa Cruz, CA). Ethyl-3,4-dephostatin, 3-Omethylfluorescein phosphate (OMFP), anti-FLAG M2 antibody, and antiFLAG M2 agarose beads were from Sigma-Aldrich (St. Louis, MO).

Purification of the six-His-tagged DUSP22 Phosphatase. DUSP22 expression plasmid was constructed in pET-28a (+) and transformed into BL21(DE3)-RIL E. coli. Recombinant DUSP22 phosphatase was induced with $0.5 \mathrm{mM}$ isopropyl$\beta$-D-thiogalactopyranoside at $37{ }^{\circ} \mathrm{C}$ for $3 \mathrm{~h}$. Cells were harvested and then lysed by sonication in $50 \mathrm{mM}$ Tris- $\mathrm{HCl}$ (pH 8), $300 \mathrm{mM} \mathrm{NaCl}, 1 \% \mathrm{NP}-40$, and $1 \mathrm{mM}$ phenylmethylsulphonyl fluoride (PMSF). The lysates were clarified at $4000 \mathrm{rpm}$ for $30 \mathrm{~min}$ at $4{ }^{\circ} \mathrm{C}$. The supernatant was applied by gravity flow to a column of Ni-NTA resin (PEPTRON, Korea). The resin was washed with $20 \mathrm{mM}$ Tris- $\mathrm{HCl}(\mathrm{pH} 8)$, $500 \mathrm{mM} \mathrm{NaCl}, 50 \mathrm{mM}$ imidazole and eluted with $20 \mathrm{mM}$ Tris- $\mathrm{HCl}$ (pH 8), $500 \mathrm{mM} \mathrm{NaCl}, 200-300 \mathrm{mM}$ imidazole. The eluted protein was dialyzed overnight against $20 \mathrm{mM}$ Tris- $\mathrm{HCl}$ (pH 8), $100 \mathrm{mM} \mathrm{NaCl}, 30 \%$ glycerol, $0.5 \mathrm{mM}$ PMSF before storage at $-80^{\circ} \mathrm{C}$.

In vitro Phosphatase Assays and Kinetic Analysis. The activity of DUSP22 phosphatase was measured using the substrate 3-Omethylfluorescein phosphate in a 96-well microtiter plate assay based on methods described previously. ${ }^{15}$ The ethyl-3,4-dephostatin and OMFP were solubilized in $\mathrm{H}_{2} \mathrm{O}$ and DMSO, respectively. All reactions were performed at a final concentration of $1 \%$ DMSO. The final incubation mixture $(150 \mu \mathrm{L})$ was optimized for enzyme activity and composed of $30 \mathrm{mM}$ Tris- $\mathrm{HCl}(\mathrm{pH} 7), 75 \mathrm{mM}$ $\mathrm{NaCl}, 1 \mathrm{mM}$ ethylenediaminetetraacetic acid (EDTA), 0.1 $\mathrm{mM}$ dithiothreitol (DTT), $0.33 \%$ bovine serum albumin (BSA) and $100 \mathrm{nM}$ of DUSP22 phosphatase. Reactions were initiated by addition of OMFP and incubated for $30 \mathrm{~min}$ at $37^{\circ} \mathrm{C}$. Fluorescence emission from product was measured with a multi-well plate reader (GENios Pro; excitation filter, $485 \mathrm{~nm}$; emission filter, $535 \mathrm{~nm})$. The reaction was linear over the time period of the experiment and was directly proportional to both enzyme and substrate concentration. Half-maximal inhibition constant $\left(\mathrm{IC}_{50}\right)$ was defined as the concentration of an inhibitor that caused a $50 \%$ decrease in the DUSP22 phosphatase activity. Half-maximal inhibition constants and best curve fit for Lineweaver-Burk plots were determined by using the curve fitting program Prism 3.0 (GraphPad Software). All experiments were performed in triplicate and were repeated at least three times.

Immunoblotting Analysis. After HEK 293 cells were cotransfected with DUSP22 WT or DACS mutant expression plasmids for $48 \mathrm{~h}$, cells were washed twice with phosphate buffered saline (PBS) buffer and lysed in PTP lysis buffer (0.5\% NP-40, 0.5\% Triton X-100, $150 \mathrm{mM} \mathrm{NaCl}, 20 \mathrm{mM}$ Tris-HCl (pH 8.0), 1 mM EDTA, 1\% glycerol, 1 mM PMSF, and $1 \mu \mathrm{g} / \mathrm{mL}$ Aprotinin) for $30 \mathrm{~min}$ at $4{ }^{\circ} \mathrm{C}$. Cleared cell lysates from centrifugation were mixed with protein sample buffer, boiled at $100^{\circ} \mathrm{C}$ for $5 \mathrm{~min}$, subjected to SDS-PAGE, and subsequently transferred onto nitrocellulose (NC) membrane. The membranes were then blocked with 5\% skim milk/TBS for $1 \mathrm{~h}$ and incubated with the appropriate primary antibodies and HRP-conjugated secondary antibodies at room temperature. Following extensive washing, protein bands were visualized using enhanced chemiluminescence (ECL) Western blotting detection reagents (Pierce, Rockford, IL, USA).

Inhibition Study. The inhibition constant $\left(K_{i}\right)$ to DUSP22 
phosphatase for the inhibitor was determined by measuring the initial rates at several OMFP concentrations for each fixed concentration of the inhibitor. The data were fitted to the following equation to obtain the inhibition constant of reversible competitive inhibitors. The slopes obtained were replotted against the inhibitor concentrations. ${ }^{16}$ The $K_{i}$ value was obtained from the slopes of these plots. ${ }^{16}$

$$
1 / \mathrm{V}=K_{m}\left(1+[\mathrm{I}] / K_{i}\right) \mathrm{V}_{\max }[\mathrm{S}]+1 / \mathrm{V}_{\max }
$$

In vivo Effect of Ethyl-3,4-dephostatin on DUSP22 Phosphatase-regulated Phospho-ERK. HEK 293 cells were transfected with FLAG-DUSP22 expression plasmid. After $48 \mathrm{~h}$ of transfection, cells were pretreated with ethyl3,4-dephostatin $(0,10,20,50 \mu \mathrm{M})$ for $3 \mathrm{~h}$. Cells were lysed in lysis buffer containing $50 \mathrm{mM}$ Tris- $\mathrm{HCl}(\mathrm{pH} 7.5), 150$ $\mathrm{mM} \mathrm{NaCl}, 1 \%$ NP-40, 0.5\% Na-deoxycholate, 1 mM EDTA, $1 \mathrm{mM}$ PMSF, $1 \mathrm{mM} \mathrm{Na} \mathrm{VO}_{4}$, and $1 \mathrm{mM} \mathrm{NaF}$. The samples were separated by SDS-PAGE, followed by Western blotting analysis.

Acknowledgments. This research was supported by the Chung-Ang University Research Scholarship Grants in 2009.

\section{References}

1. Camps, M.; Nichols, A.; Arkinstall, S. FASEB J. 2000, 14, 6.
2. Franklin, C. C.; Kraft, A. S. J. Biol. Chem. 1997, 272, 16917.

3. Bost, F.; Aouadi, M.; Caron, L.; Binetruy, B. Biochimie 2005, 87, 51.

4. Davis, R. J. Cell 2000, 103, 239.

5. Johnson, G. L.; Lapadat, R. Science 2002, 298, 1911.

6. Alonso, A.; Sasin, J.; Bottini, N.; Friedberg, I.; Osterman, A.; Godzik, A.; Hunter, T.; Dixon, J.; Mustelin, T. Cell 2004, 117, 699.

7. Patterson, K. I.; Brummer, T.; O'Brien, P. M.; Daly, R. J. Biochem. J. 2009, 418, 475

8. Fischer, E. H.; Charbonneau, H.; Tonks, N. K.Science 1991, 253, 401.

9. Suzuki, T.; Hiroki, A.; Watanabe, T.; Yamashita, T.; Takei, I.; Umezawa, K. J. Biol. Chem. 2001, 276, 27511.

10. Watanabe, T.; Suzuki, T.; Umezawa, Y.; Takeushi, T.; Otsuka, M.; Umezawa, K. Tetrahedron 2000, 56, 741.

11. Alonso, A.; Merlo, J. J.; Na, S.; Kholod, N.; Jaroszewski, L.; Kharitonenkov, A.; Williams, S.; Godzik, A.; Posada, J. D.; Mustelin, T. J. Biol. Chem. 2002, 277, 5524.

12. Aoyama, K.; Nagata, M.; Oshima, K.; Matsuda, T.; Aoki, N. J. Biol. Chem. 2001, 276, 27575.

13. Chen, A. J.; Zhou, G.; Juan, T.; Colicos, S. M.; Cannon, J. P.; Cabriera-Hansen, M.; Meyer, C. F.; Jurecic, R.; Copeland, N. G.; Gilbert, D. J.; Jenkins, N. A.; Fletcher, F.; Tan, T. H.; Belmont, J. W. J. Biol. Chem. 2002, 277, 36592.

14. Shen, Y.; Luche, R.; Wei, B.; Gordon, M. L.; Diltz, C. D.; Tonks, N. K. Proc. Natl. Acad. Sci. USA 2001, 98, 13613.

15. Tierno, M. B.; Johnston, P. A.; Foster, C.; Skoko, J. J.; Shinde, S. N.; Shun, T. Y.; Lazo, J. S. Nat. Protoc. 2007, 2, 1134.

16. Shi, Z.; Tabassum, S.; Jiang, W.; Zhang, J.; Mathur, S.; Wu, J.; Shi, Y. Chembiochem. 2007, 8, 2092. 